\title{
BATERIA PARA AVALIAÇÃO NEUROPSICOLÓGICA DE ADULTOS COM EPILEPSIA
}

\author{
Élia Baeta
}

Resumo A observação neuropsicológica faz parte da avaliação do doente epiléptico. O objectivo deste trabalho é a normalização de uma bateria de avaliação para doentes com epilepsia focal de origem temporal ou frontal. Cento e sessenta indivíduos, sem patologia neurológica, ou psiquiátrica, foram submetidos a avaliação neuropsicológica, através de um conjunto de testes com reconhecida capacidade de detecção de disfunções focais de natureza comicial. Os resultados são apresentados de acordo com os grupos etários e graus de escolaridade. Verifica-se que existem diferenças no desempenho nas provas verbais e não verbais, relacionadas com as características demográficas: escolaridade, idade e sexo. Assim, é demonstrada a importância da normalização de qualquer bateria. Testes que, em outras culturas, não demonstram variabilidade sociodemográfica foram, na nossa população, sensíveis às diferença de sexos e estratos etários e culturais.

Palavras-chave Avaliação neuropsicológica, epilepsia, testes.

\section{Introdução}

A epilepsia é uma doença comum que afecta todos os grupos etários. O uso do exame neuropsicológico como complemento de avaliação tem vindo a expandir-se. A integração dos dados obtidos com a clínica e com resultados de outros métodos complementares proporciona orientação diagnóstica e prognóstica (Milner, 1967; Rausch et al., 1998). Contribui, por exemplo, para a confirmação da existência e localização do foco epileptogéneo (Jones-Gotman, 1991), razão pela qual tem sido usado na avaliação do doente candidato a tratamento cirúrgico. Pode também determinar a ocorrência de deterioração cognitiva, ou os efeitos de medicamentos antiepilépticos.

Nas crianças e adolescentes, a epilepsia pode produzir problemas de ajustamento social que, por vezes, são confundidos com baixo nível intelectual. São comuns queixas cognitivas, sendo as mais frequentes relativas à memória. Um exame desta natureza deve ser capaz de evidenciar alteração cognitiva e assimetrias no sentido do defeito e também nos aspectos positivos (desempenhos na média ou superiores). O perfil permite orientar o indivíduo na elaboração de 
estratégias compensadoras. Por sua vez, os adolescentes e adultos jovens podem ser dirigidos para áreas profissionais que lhes serão vantajosas.

A literatura é rica em trabalhos que têm mostrado o papel primordial do exame das funções nervosas superiores. No entanto, o seu uso está pouco divulgado, restringindo-se aos grandes centros ou instituições que realizam tratamento cirúrgico. Os métodos usados são muito diferentes entre os centros (Jones-Gotman, 1991; Rausch et al., 1998), limitando a comparação da análise dos resultados. Além da diversidade dos métodos, muitos autores focam os testes em funções particulares, não permitindo uma avaliação compreensiva do doente. As baterias de avaliação devem examinar funções hemisféricas direitas e esquerdas, áreas anteriores e posteriores. A sensibilidade dos testes deve ser elevada porque a extensão da disfunção é frequentemente mínima. Devem ser capazes de detectar a integridade de funções cerebrais gerais e focais através da avaliação de linguagem, memória, atenção e concentração, capacidades visuoespaciais e construtivas, flexibilidade mental, capacidade de resolução de problemas e capacidades sensoriais e motoras (Rausch et al., 1998). No nosso país nunca foi elaborada, nem normalizada, uma bateria que se adequasse à avaliação de doentes com epilepsia.

Na realidade portuguesa, um dos maiores problemas que afecta a avaliação neuropsicológica destes doentes é a falta de testes e normas adequadas. Este problema assume particular importância quando, sendo desejável uniformizar os métodos de medição, se tenta usar testes de outras culturas na camada menos diferenciada da nossa população. A normalização dos dados dos testes é, pois, fundamental e imprescindível para a determinação da influência dos factores sociodemográficos e interpretação dos resultados (Jones-Gotman, 1991; Saykin et al., 1995).

A bateria de testes que apresentamos foi elaborada para avaliação específica no domínio do funcionamento cognitivo de doentes com epilepsia focal temporal ou frontal. Possui características próprias que permitem o uso com objectivos clínicos e de investigação. É natural que, para avaliação de situações particulares em doentes epilépticos, deva ser modificada ou expandida, ou que outra bateria tenha que ser construída.

Pretende-se também determinar o efeito das mais importantes variáveis demográficas (idade e escolaridade) na resolução dos testes e estratificar os dados de acordo com essas variáveis.

\section{Método}

\section{População}

A população inicial consistiu em 200 indivíduos portugueses, saudáveis, recrutados da comunidade através de apelos aos familiares e amigos de doentes 
Quadro 1 Caracteristicas sociodemograticas da populaçlo estudada

\begin{tabular}{lcc}
\hline & Media (DP) & Intervalo \\
\hline Idade & $29,0+8,8$ & $15-45$ anos \\
Educaçalo & $11,1+3,7$ & $4-19$ anos \\
\hline & $\mathrm{N}$. & \\
\hline Homens & $52(32,5 \%)$ & \\
Mulheres & $108(67,5 \%)$ & \\
\hline Dextros & $160(100 \%)$ \\
\hline
\end{tabular}

com epilepsia, a familiares de outros doentes e, em pequeno número, a familiares ou elementos do pessoal do hospital.

Foram excluídos indivíduos com história de doença neurológica ou dificuldades de aprendizagem, doença ou medicação psiquiátrica, doença de outro órgão ou sistema com eventual repercussão no funcionamento cognitivo, abuso de álcool ou drogas. Indivíduos aos quais faltavam dados demográficos ou com história clínica duvidosa não foram aceites. A amostra final ficou constituída por 160 indivíduos.

Tinham todos entre 15 e 45 anos, eram todos dextros (lateralidade determinada pelo Edinburgh Inventory (Oldfield, 1971)) e nenhum era iletrado. A tabela 1 mostra as características sociodemográficas da população.

\section{Procedimentos}

Para a elaboração da bateria de testes neuropsicológicos foram escolhidos e adaptados testes com reconhecida capacidade de avaliação de sistemas cerebrais regionais. Memória e aprendizagem (verbal e visual), linguagem (recepçăo $\mathrm{e}$ expressão), atenção, raciocínio não verbal, processamento cognitivo visual espacial e verbal. Os testes incluídos estão representados no quadro 2.

Os testes foram administrados numa sequência fixa, por examinadores treinados e com modo padronizado de aplicação. $\mathrm{O}$ tempo de aplicação da bateria foi, em média, de duas horas. Raramente foi necessário fazer intervalo.

\section{Descrição dos Testes}

Repetição de dígitos

Esta prova é um subteste da prova Escala de Memória de Wechsler (EMW) forma I (Weschler, 1945) e consta de dois subtestes. Um em que o individuo repete os algarismos por ordem directa (DD) e um em que estes são repetidos por ordem inversa 
Quadro 2 Bateria neuropsicológica

Atencso

- Repeticlo de digitos (5)

- Controlo mental (5)

Processamento e atenclo visual

- Toulouse Pieron (6)

- Stroop Colour-Word Test (9)

- Trail Making Test (10)

Alternaincia de taretas

- Sequencias Grafomotoras (11) (12)

- Wisconsin Card Sorting Test (13)

Linguagem

- Nomeacalo oral semántica (11) (12)

- Token Test modificado (16) (17) (18)

- Nomeaclo de Objectos

- Fluencia Verbal Escrita (19)

- Leitura de tento (16) (17) (18)

Capacidades etpaciais

- Orientaclo de Linhas (21)

- Copia de um cubo (2a)

Memoria e aprendizagem verbal

- Memoria Iogica (7) (23) (24)

- Aprendizagem Associativa (23) (24)

- California Verbal Learning Test (26)

Aprendizagem imediata

Aprendizagem apos curto tempo de intervalo sem e com ajuda semantica

Aprendizagem após longo tempo de latência sem e com ajuda

Peconhecimento

Memoria visual

- Teste de memoria visual - (24) (27) (28)

Raciocinio nso verbal

- Matrizes de Raven, forma abreviada para advatos (30)

Testes de lateralidade

- Finger Tapping (10) (31)

- Grooved Pegboard (32)

- Forca de Preensalo (31)

- Teste ABC visual (33)

(DInv). São cotados separadamente. O primeiro subteste avalia sobretudo a quantidade de informação que cada um consegue reter, num dado momento, através da memória de trabalho. Por ordem inversa não só é avaliada a capacidade de atençăo dividida como também a de manipulação mental. A realizaçāo de ambas as tarefas é largamente dependente do lobo frontal (Spreen \& Strauss, 1987; Lezak, 1995).

\section{Controlo Mental (C Mental)}

É um subteste da EMW em que, para a realizaçầo das três tarefas de que é composto (contar por ordem inversa de 20 até 1; evocar o alfabeto e contar de $3 \mathrm{em} 3$ o mais rapidamente possível), é necessário estarem mantidas algumas capacidades, tais 
como atenção, concentração e capacidade de planeamento. A classificação é feita de acordo com as normas da EMW.

Teste de Toulouse Pieron

É um teste muito pouco conhecido, embora de grande utilidade na avaliação da atenção mantida por longos períodos de tempo, dependente do lobo frontal. Provavelmente a baixa popularidade é devida à longa duração (10 minutos). É um teste de barragem em que são testados o processamento visual e a capacidade de activação e inibição de respostas rápidas. Numa folha de tipo A3 existem 25 filas de 40 itens. Em cada fila horizontal estão dispersos 15 carateres-alvo que o examinando deve assinalar. São contados os alvos assinalados correctamente (C), os falsos positivos (FP) e os falsos negativos (FN) (Aria, 1958). São medidos dois índices: RT (rendimento de trabalho) e ID (índice de dispersão), que se calculam do seguinte modo:

$$
\mathrm{RT}=\mathrm{C}-(\mathrm{FP}+\mathrm{FN}) \quad \mathrm{ID}=(\mathrm{FP}+\mathrm{FN}) / \mathrm{C}
$$

Baixos desempenhos em RT podem corresponder a, inatenção, lentificação geral da resposta; em ID a aumento da distractibilidade. A perturbação da alternância de tarefas, negligência unilateral, dificuldade no processamento de informação e integração motora atinge habitualmente os dois índices.

\section{Stroop Colour Test}

A forma que usamos é uma versão reduzida do original apenas com duas páginas de apresentação. Em cada página estão impressas quatro colunas de palavras (nomes de 4 cores), cada uma com 28 elementos. O nome de uma cor (preto, vermelho, verde ou azul) está sempre impresso numa cor diferente, ou seja, existe dissociação de nomes e de cores. Na primeira página (C) é pedido para serem lidas as palavras o mais rapidamente possível. Na segunda página $(C W)$ é pedido para nomear a cor em que cada palavra está impressa. Em ambas as folhas o tempo é cronometrado. Cada erro é corrigido imediatamente com paragem de progressão na prova. São cotados o tempo de realização (em segundos) e os erros em cada subteste (que são tidos em conta só se o tempo estiver prolongado) (ERRO-C; ERRO CW). Mede a capacidade de cada pessoa modificar a sua percepção estabelecida de acordo com as alterações necessárias em suprimir a resposta habitual em favor de uma não usual (Golden, 1978). Avalia portanto a capacidade de resistência à interferência de uma tarefa dissociativa, capacidade que é dependente do lobo frontal.

\section{Trail Making Test (formas A e B)}

É composto por duas formas ou subtestes, A e B. Na folha A requer-se a ligação de 25 números por uma linha e por ordem crescente. Na forma B pretende-se a ligação 
alternada de letras e números (Reitan \& Wolfson, 1985) dispersos na folha. A realização de ambas as formas é cronometrada e a prova é interrompida para correcção dos erros. Além do tempo de realização de cada uma das formas, tem-se em conta o número de erros (ERRO A e ERRO B).

É altamente vulnerável aos efeitos de lesão cerebral e para a sua realização é necessária uma boa capacidade de pesquisa visual, atenção, concentração visuomotora e flexibilidade mental.

\section{Sequências Grafomotoras (S Luria.)}

Os dois subtestes usados são derivados da Bateria de Luria (Luria, 1965; Christensen, 1975) e são sequências motoras que implicam a activação alternada de diferentes padrões motores. Em caso de lesão frontal, existe perturbação na alternância activação/inibição daqueles padrões, desencadeando perseveração. De cada vez pede-se para copiar três linhas sequenciais. O resultado é qualitativo: positivo quando o indivíduo persevera e negativo se isso não se verifica.

\section{Wisconsin Card Sorting Test (WCST)}

Neste teste existem quatro cartões modelo: um com um triângulo vermelho, um com duas estrelas verdes, um com três cruzes amarelas e um com quatro bolas azuis. O objectivo é emparceirar dois baralhos (cada um com 64 cartões), com os cartões modelo pelas três lógicas possíveis (forma-F, cor-C e número-N) (Heaton, 1981). Além da interpretação correcta das lógicas que governam o teste é necessário que, sem aviso, o examinando consiga abandonar uma lógica em vigor para descobrir, o mais rapidamente possível, a nova que o examinador escolheu. Na versão que usámos são realizadas quatro sequências $(C, F, N, C)$. É contado o número de respostas correctas e o número de erros, particularmente de perseverações, que em número aumentado evidenciam disfunção do lobo frontal.

\section{Nomeação Oral Semântica}

Das variadíssimas versões de nomeação por categoria semântica escolhemos dois testes: num pede-se para evocar artigos de comer (IVO1) e noutro nomes de animais (IVO2). O resultado é o número de palavras correctamente evocadas ao fim de um minuto, excluindo as perseverações. Testa linguagem e memória semântica. A maior parte dos trabalhos publicados em que é empregue esta tarefa relacionam-na com o lobo frontal esquerdo (Lezak, 1995; Spreen \& Strauss, 1987). De acordo com Jokeit et al. (1988) (14), a nomeação de nomes de animais é sensível a lesões do hemisfério esquerdo, e é também afectada por um foco temporal esquerdo, não quando este se localiza no lobo temporal direito.

\section{Token Test (TT)}

A versão do teste usada é algo diferente da original, proposta por De Renzi e 
Vignolo (1962). Trata-se da adaptação à língua portuguesa de uma versão reduzida de 22 ordens e é a usada na Bateria de Afasia de Lisboa (Damasio, 1973; Castro-Caldas, 1979; Ferro, 1986). É um bom teste de compreensão auditiva, portanto sensível a distúrbios das áreas têmporo-parietais de linguagem, mas o seu desempenho pode ser interferido por alterações da atenção. A cotação é efectuada segundo as normas habituais.

\section{Nomeação de Objectos}

Pede-se a nomeação por confrontação visual de 20 objectos de uso habitual nas actividades diárias. É um teste sensível às lesões do hemisfério dominante para a linguagem, sobretudo às disfunções de áreas relacionadas com a produção verbal.

Fluência Verbal Escrita (FVE)

Existem várias formas deste teste. Escolhemos uma em que é pedido para escrever o máximo de palavras (não derivadas) começadas pela letra S. A duração da prova é de cinco minutos. Não são aceites palavras escritas em outras línguas para além da portuguesa. Avalia a capacidade de fluência fonológica que está relacionada com a função do hemisfério dominante para a linguagem (Lezak, 1995). A cotação é o número de palavras escritas, excluindo as palavras da mesma família e as perseverações.

\section{Leitura de Texto}

A prova de leitura é a usada na Bateria de Afasia de Lisboa (Castro Caldas, 1979; Ferro, 1986) em que, para além de eventuais paralexias, se anota a compreensão do texto em resposta a seis questões relacionadas com o seu conteúdo.

Orientação de Linhas (TOL)

O objectivo deste teste é emparceirar segmentos de recta com um modelo, cartãoresposta, em que estão representados segmentos mais longos e com várias orientações. Este teste é sensível a lesões do hemisfério cerebral direito (Benton et al.,1983), sobretudo às localizadas em áreas posteriores. A nota final é o número de pares em que a orientação espacial dos dois segmentos foi correcta.

Cópia de um Cubo (CUBO)

Esta prova foi escolhida para avaliação sumária das capacidades visuoconstrutivas. Pretende-se que o sujeito desenhe um cubo, por cópia de um modelo representado na folha de prova. A cotação foi modificada em relação ao original, sendo atribuído um ponto por cada aresta correctamente representada. É retirado um ponto se as dimensões das arestas forem inadequadas ou se houver um número de arestas superior ao do modelo. 


\section{Memória Lógica}

Adoptámos a forma I da EMW modificada (Russel, 1975; Ivnik, 1987; Spreen \& Strauss, 1987). São lidas duas histórias e após cada uma delas é pedido ao sujeito para recordar o maior número de ideias possíveis (MLOG). O resultado corresponde ao número de ideias obtido em cada história, segundo as regras de cotaçăo da EMW.

Após um intervalo de 30 minutos pede-se de novo para serem recordadas o maior número de ideias possíveis (MLOGL). A cotação é semelhante à da forma imediata. É um teste que se baseia no processamento de informação sintáctico-semântica. Examina a memória episódica, mas é também influenciada pela capacidade de atenção e concentração.

Aprendizagem Verbal Associativa

Esta prova, mais conhecida por Prova de Pares de Palavras (PP), requer que o indivíduo dê atenção a uma lista de pares de palavras associadas e que, após a leitura da lista, evoque a segunda palavra de cada par quando lhe é fornecida a primeira (PP). São feitas três tentativas de aprendizagem da lista.

Tal como na prova anterior, foram adoptadas modificaçôes em relação à versão clássica da forma I da EMW. Sem qualquer aviso, meia hora depois é de novo pedido ao sujeito para evocar a palavra certa em resposta à palavra-estímulo (PPL). Nos pares em que a resposta é errada tenta-se a escolha múltipla com três opçōes: uma certa, uma intrusăo e uma transposição. As cotaçōes seguem as normas dos autores originais (Russel, 1975; lvnik, 1987). Avalia-se assim a memória verbal associativa e a capacidade de aprendizagem dependentes da função do lobo temporal.

\section{California Verbal Learning Test (CVLT)}

Uma lista ("de 2." feira") de 16 palavras pertencentes a quatro categorias semânticas diferentes é apresentada e evocada cinco vezes. Em seguida é lida uma outra lista ("de 3." feira"). Esta é constituída também por 16 palavras de quatro categorias semânticas e duas delas são iguais às da lista anterior. Após a evocação livre da lista de 3." feira é pedida a evocaçăo livre e com ajuda semântica da lista de 2 ." feira. Ao fim de 20 minutos de intervalo é pedida a evocaçăo livre e com ajuda semântica da lista de $2{ }^{*}$ feira. Por fim é pedido o reconhecimento das 16 palavras da lista de 2. " feira entre 44 palavras (16 da lista de $2{ }^{*}$ feira e as restantes da lista de 3." feira e sem qualquer relaçăo com a prova).

Apesar do grande número de índices que se podem obter deste teste apenas retirámos oito. Três deles medem a capacidade de aprendizagem através da repetição dos estimulos: desempenho na 1." evocação livre da lista de 2. "feira (C1)e que mede a capacidade de codificação da informação; desempenho na 5 , evocaçăo da lista de 2."feira (C5); soma dos totais das cinco evocaçôes (Ctotal) relacionadas com a aprendizagem. A evocação livre (CIC) e com ajuda semântica (CICAS) da 
lista de 2. " feira após interferência da lista de $3 .{ }^{\text {a }}$ feira; a evocação da lista de $2 .{ }^{\text {a }}$ feira após 20 minutos de intervalo, livre (CIL) e com ajuda semântica (CILAS); o desempenho na prova de reconhecimento (REC) determina a capacidade de retenção da informação.

\section{Reprodução Visual}

Este é um subteste da forma I da EMW, que avalia a memória visual, e em que é pretendido o desenho numa folha de papel, após a sua apresentaçăo por dez segundos. Os desenhos a representar são quatro e estão divididos por três cartões (MV). Cada desenho é classificado segundo as regras da WMS. A soma das cotações dos quatro desenhos é o resultado final.

Após 30 minutos, são de novo evocados, por desenho, os modelos apresentados anteriormente (MVL). Se isso não for conseguido é feita uma tentativa de reconhecimento de cada desenho esquecido entre cinco opções. $\mathrm{O}$ resultado final é a soma da cotação em cada desenho evocado (segundo as regras da EMW) e de 1/2 do valor total para cada modelo não evocado mas reconhecido. Esta modificação da apresentação e cotaçăo do teste melhorou a sua sensibilidade às disfunções do lobo temporal direito (Minden et al., 1988; Trahan et al., 1988).

\section{Matrizes de Raven, forma abreviada para adultos (MRaven).}

Além de ser muito popular, segundo Lezak (1995), não requer diferenciação especial em informação visuoespacial, também năo requer verbalização e por isso tornou-se um teste breve de avaliaçăo de capacidade intelectual (Raven, 1938). A forma abreviada que adoptámos já foi testada numa populaçāo portuguesa de crianças e adolescentes (30), e consiste na apresentação de 30 cartōes agrupados em cinco séries. Acada modelo é removida uma porção que se encontra entre as opçōes de resposta (6 ou 8). Os resultados relacionam-se com as capacidades visuoconstrutivas (Lezak, 1995), sendo por isso mais sensiveis a disfunçōes do hemisfério direito.

\section{Finger Tapping Test"}

Com a mão em cima da mesa e o indicador numa tecla ligada a um contador, o examinando deve, durante 15 segundos, carregar nela tantas vezes quantas possível. Inicia-se com a mão preferencial e testa-se a mão dominante alternadamente, por três vezes. Ovalor é a média das três tentativas. É um bom instrumento, não só das diferenças de destreza motora como de velocidade motora.

\section{Grooved Pegboard}

O objectivo é o doente encaixar muito rapidamente pequenos pregos em 25 buracos dispostos em cinco filas num pequeno tabuleiro. Cada prego tem uma 
aresta que deve encaixar na ranhura do buraco. A orientação da ranhura é diferente de buraco para buraco. Cada mão é testada separadamente e o tempo é cronometrado. Fazem-se três tentativas para cada mão. Esta é uma boa e rápida medida de coordenação motora manual.

Força de Preensão

Inicia-se com a mão dominante que tem mais força. Pede-se ao sujeito que aperte a pega de um dinamómetro com o membro esticado e afastado do tronco. Antes de iniciar a prova deve-se proporcionar uma tentativa para prática. A medição da força é obtida pela média de três tentativas nos dois membros superiores.

Teste A-B-C Visual

O teste A-B-C tem por objectivo detectar rapidamente o olho dominante. Com a ajuda de três visores o examinando nomeia o desenho ou diferencia tonalidades de dez cartões apresentados a três metros pelo examinador. Através da parte mais estreita do visor o examinador vê qual o olho que fixa o cartão.

\section{Resultados}

Os resultados dos testes foram registados na base de dados do programa de estatística SPSS8.0 (1997). A análise estatística foi realizada no mesmo programa.

Os testes de dominância motora (Finger Tapping Test, Grooved Pegboard, força de preensão) e ocular (teste $\mathrm{ABC}$ ) não foram submetidos a avaliação estatística, por apenas interessarem como forma de testar a dominância cerebral, medindo-a no paciente.

O WCST não foi alvo de tratamento estatístico por, na análise preliminar, se verificar que em todos os grupos houve muitos individuos que não conseguiram obter pontuação.

\section{Análise de variância}

A análise dos dados envolveu, numa primeira fase, o estudo da influência das características demográficas, sexo, idade e escolaridade, no desempenho de cada teste.

Os testes de nomeação de objectos e leitura de texto não foram submetidos a esta análise por não apresentarem variância. No caso da nomeação de objectos, todos os elementos obtiveram a pontuação máxima $(20 / 20)$, assim como na prova de compreensão de texto $(6 / 6)$. 
Quadro 3 Influêncla das variaveis escolaridade, idade e sexo no desempenho das provas da Bateria Neuropsicologica para Epilepsia

\begin{tabular}{|c|c|c|c|c|c|c|c|c|c|c|c|c|c|c|}
\hline \multirow[t]{3}{*}{ Testes } & \multicolumn{14}{|c|}{ Varihveis } \\
\hline & \multicolumn{2}{|c|}{ Escolaridade } & \multicolumn{3}{|c|}{ Schefle } & \multicolumn{2}{|c|}{ Idade } & \multicolumn{3}{|c|}{ Schetle } & \multicolumn{4}{|c|}{ Sexo } \\
\hline & F ratio & F prob. & 1 & 2 & 3 & F ratio & Fprob. & 1 & 2 & 3 & F ratio & F prob. & $F$ & $M$ \\
\hline AT & 4.9003 & 0,0097 & 3 & & & 5,4720 & 0.0058 & & & 1.2 & - & - & & \\
\hline id & - & - & & & & 5.0797 & 0,0082 & $\mathbf{3}$ & & & - & - & & \\
\hline MLOG & 10,3845 & 0.0001 & 3 & & & - & - & & & & - & - & & \\
\hline MLOGL & 9,3874 & 0,0002 & 3 & 3 & & - & - & & & & - & - & & \\
\hline$p p$ & 7,9032 & 0.0007 & 3 & & & 5.3911 & 0.0062 & & & 1.2 & - & $=$ & & \\
\hline PPL & 8.6544 & 0,0004 & 3 & & & - & - & & & & - & - & & \\
\hline MV & 11,4753 & 0,0000 & 2.3 & & & 4.4214 & 0,0149 & & & 1 & - & - & & \\
\hline $\mathrm{MV}$ & 3.3940 & 0.0382 & 3 & & & - & - & & & & - & - & & \\
\hline Dinv & 6.499 & 0.0024 & 3 & & & - & - & & & & - & - & & \\
\hline STROOP & - & - & & & & 4,9683 & 0,0091 & & & 1.2 & - & - & & \\
\hline $\mathrm{cW}$ & & & & & & & & & & & & & & \\
\hline Not & - & - & & & & - & - & & & & - & - & & \\
\hline NOR & 3.9266 & 0.0234 & 3 & & & - & - & & & & - & - & & \\
\hline FVE & 14.6422 & 0.0000 & 2.3 & & & - & - & & & & - & - & & \\
\hline Trall A & 3,2819 & 0,0424 & & & 1 & - & - & & & & - & - & & \\
\hline TAAIL B & 11.0791 & 0.0001 & & 1 & $i$ & - & - & & & & - & - & & \\
\hline ERAOB & 4.9247 & 0,0095 & & $i$ & 1 & - & - & & & & - & - & & \\
\hline$\pi$ & 9.5955 & 0,0002 & 2.3 & & & - & - & & & & - & - & & \\
\hline CVLT.5 & - & - & & & & - & - & & & & 13,3943 & 0.0004 & - & \\
\hline CVLT-ic & - & - & & & & - & - & & & & 6,1057 & 0.0154 & - & \\
\hline CVLTticas & - & - & & & & - & - & & & & 5,7681 & 0.0185 & - & \\
\hline chrtal & - & - & & & & - & - & & & & 5,0190 & 0.0276 & . & \\
\hline CVIT-total & 5,7960 & 0,0044 & 3 & 3 & & - & $=$ & & & & 10,6244 & 0.0016 & - & \\
\hline cubo & & & & & & 3.5577 & 0.0328 & 3 & & & - & - & & \\
\hline M RAVEN & 21,1490 & 0,0000 & 2.3 & & & 3,3921 & 0.0363 & ; & & & 4,4924 & 0.0960 & & - \\
\hline Ta & 4.4508 & 0.0145 & 3 & & & - & - & & & & 9.6861 & 0.0025 & & - \\
\hline
\end{tabular}

Notas: escolaridade: grupo 1- $<=9 a n o s ;$ grupo 2-10-12 anos; grupo 3- $>=13$ anos. Idade: grupo 1- 15-25anos; grupo 2-26-35 anos; grupo3-36-45 anos: Sexo: F-feminino; M-masculino.

As notas obtidas em cada teste foram comparadas através do método ANOVA. Para evitar erros de tipo I escolhemos a análise post-hoc de Scheffe, por permitir a comparação de resultados em grupos de diferente dimensão. Foram usados três grupos de escolaridade $(<=9 ; 10-12 ;=>13$ anos) e três grupos de idade (15-25; 26-35; 36-45 anos).

Para testes de valores de capacidades, os valores mais altos correspondem ao melhor desempenho. Em provas com contagem de tempos e erros, como no Stroop Test ou no Trail Making Test, os valores mais altos correspondem a piores resultados e a pior desempenho.

A escolaridade é o factor que mais interfere com a realização das provas. Os indivíduos com escolaridade superior diferem significativamente dos que têm apenas a escolaridade básica. Todas as provas da EMW são sensíveis à escolaridade, assim como o indice CVLT total. Os testes de atençăo sustida (índices RT e ID do Teste de Toulouse Pieron) e os que implicam capacidades visuoespaciais e visuoperceptivas (Trail Making Test, Matrizes de Raven e LOT) sofrem a mesma influência. Também nos testes de iniciativa verbal oral e escrita se obtêm melhores resultados se o grau de escolaridade for superior. 
A idade apresenta uma relação inversa com o desempenho nas Matrizes de Raven e nas provas de memória (reprodução visual e aprendizagem verbal associativa). Os mais novos são mais rápidos nos testes de atenção sustida e interferida (índices RT, ID e teste Stroop CW). Apesar de a capacidade práxica construtiva ser muito estável, nesta amostra o grupo com mais idade desenha melhor o cubo por cópia.

Como está descrito, as mulheres têm um desempenho superior ao dos homens no teste CVLT (Delis et al., 1987). O sexo masculino evidencia melhor desempenho na prova de orientação espacial de linhas (TOL) e MRaven.

\section{Normas estratificadas por idade e escolaridade}

Uma vez que existe marcada influência dos factores demográficos nos resultados dos testes,é essencial que os valores-padrão sejam estratificados. Como na variável sexo os grupos são de grandeza diferente, os valores foram estratificados apenas para as variáveis idade e escolaridade.

Foram usados três níveis de idade: 15-25, 26-35e 36-45 anos. Os três grupos de escolaridade correspondem aos níveis mais habituais na população portuguesa: 9 anos ou menos; 10-12 anos; 13 anos ou mais. Por falta de elementos num dos grupos, criou-se um único grupo com os indivíduos com mais de 26 anos de idade $e$ com escolaridade entre os 10-12 anos.

O quadro 4 mostra as características demográficas de cada grupo. O quadro 5 mostra os dados de normalização estratificados por idade e grau de escolaridade.

Quadro 4 Caracteristicas demográficas dos subgrupos divididos por idade e grau de escolaridade

\begin{tabular}{lcccc}
\hline $\begin{array}{l}\text { Grupo por idade } \\
\text { e escolaridade }\end{array}$ & $\begin{array}{c}N^{2} \text { de } \\
\text { elementos }\end{array}$ & $\begin{array}{c}\text { Sexo } \\
(\mathrm{f} / \mathrm{m})\end{array}$ & $\begin{array}{c}\text { Idade } \\
\text { (media / dp) }\end{array}$ & $\begin{array}{c}\text { Escolaridade } \\
\text { (media / dp) }\end{array}$ \\
\hline $\begin{array}{l}15-25 \\
<=9\end{array}$ & 20 & $11 \mathrm{M} / 9 \mathrm{~F}$ & $19,2+2,6$ & $7,6+1,5$ \\
$\begin{array}{l}26-35 \\
<=9\end{array}$ & 21 & $7 \mathrm{M} / 14 \mathrm{~F}$ & $31,6+2,1$ & $7,1+1,6$ \\
$\begin{array}{l}36-45 \\
<=9\end{array}$ & 20 & $3 \mathrm{M} / 17 \mathrm{~F}$ & $40,5+3,2$ & $6,7+2,0$ \\
$\begin{array}{l}15-25 \\
10-12\end{array}$ & 25 & $5 \mathrm{M} / 20 \mathrm{~F}$ & $19,1+2,1$ & $11,5+0,7$ \\
$\begin{array}{l}26-45 \\
10-12\end{array}$ & 21 & $12 \mathrm{M} / 9 \mathrm{~F}$ & $35,2+6,2$ & $11,2+0,7$ \\
$\begin{array}{l}>=13 \\
26-35\end{array}$ & 21 & $3 \mathrm{M} / 18 \mathrm{~F}$ & $22,7+1,9$ & $14,9+0,9$ \\
$>=13$ & 20 & $5 \mathrm{M} / 15 \mathrm{~F}$ & $29,7+3,1$ & $15,7+1,6$ \\
$36-45$ & 12 & $6 \mathrm{M} / 6 \mathrm{~F}$ & $41,8+3,0$ & $15,5+1,3$ \\
\hline$=13$ & & & & \\
\hline
\end{tabular}


Quadro 5 Valores normais estratificados por idade e escolaridade

\begin{tabular}{|c|c|c|c|c|c|c|c|c|c|c|}
\hline \multirow{3}{*}{$\begin{array}{l}\text { Idade } \\
\text { Escolaridade } \\
\text { Testes }\end{array}$} & \multirow{2}{*}{\multicolumn{2}{|c|}{$\begin{array}{l}15-25 \\
<=9\end{array}$}} & \multirow{2}{*}{\multicolumn{2}{|c|}{$\begin{array}{l}26-35 \\
<=9\end{array}$}} & \multirow{2}{*}{\multicolumn{2}{|c|}{$\begin{array}{l}36-45 \\
<=9\end{array}$}} & \multirow{2}{*}{\multicolumn{2}{|c|}{$\frac{15-25}{10-12}$}} & \multirow{2}{*}{\multicolumn{2}{|c|}{$\begin{array}{l}26-45 \\
10-12 \\
\end{array}$}} \\
\hline & & & & & & & & & & \\
\hline & Média & DP & Média & DP & Media & DP & Media & DP & Media & DP \\
\hline C. MENTAL & 5,61 & 1,49 & 6,71 & 1,84 & 6,26 & 1,62 & 6,32 & 1,30 & 7,00 & 1,56 \\
\hline RT & 180,76 & 53,93 & 191,95 & 60 & 161,31 & 53,84 & 213,07 & 44,49 & 236,95 & 63,41 \\
\hline utolD & 15,61 & 11,43 & 18,35 & 9,89 & 23,69 & 19,41 & 13,63 & 9,28 & 11,50 & 7,56 \\
\hline MLOG & 9.81 & 2,79 & 9,83 & 2,80 & 9,81 & 2,91 & 12,12 & 2,92 & 11,58 & 3,09 \\
\hline MLOGL & 8,92 & 3,06 & 8,71 & 2,92 & 9,05 & 3,00 & 11,17 & 3,38 & 10,27 & 3,36 \\
\hline PP & 16,09 & 4,55 & 17,28 & 3,22 & 15,26 & 4,59 & 18,98 & 1,66 & 17,60 & 3,17 \\
\hline PPL & 9,19 & 1,04 & 9,31 & 1,03 & 9,31 & 0,74 & 9,91 & 0,23 & 9.52 & 0,61 \\
\hline MV & 12,35 & 2,18 & 10,85 & 2,81 & 9,97 & 2,42 & 12,78 & 1,68 & 12,95 & 2,13 \\
\hline MVL & 12,07 & 2,30 & 10,19 & 3,75 & 9,31 & 2,90 & 12,42 & 1,93 & 11,93 & 2,56 \\
\hline DD & 5,14 & 0,96 & 5,76 & 1,41 & 5,10 & 1,19 & 6.28 & 1,32 & 6.08 & 1,38 \\
\hline Dinv & 4,23 & 1,09 & 4,42 & 1,63 & 4,15 & 0,95 & 5.28 & 1,27 & 4,66 & 1,16 \\
\hline STAOOP C & 63,19 & 19,26 & 55,52 & 14,21 & 65,47 & 23,01 & 59,67 & 9,74 & 55,50 & 7,36 \\
\hline ERRO C & $0-2$ & & $0-7$ & & 0.1 & & 0.2 & & $0-1$ & \\
\hline STROOP CW & 138,5 & 23,96 & 138,19 & 33,49 & 146,66 & 23,43 & 130,10 & 26,54 & 131,29 & 29,54 \\
\hline ERRO CW & 0.7 & & $0-8$ & & 0.6 & & 0.10 & & $0-8$ & \\
\hline IVO1 & 19,38 & 5,68 & 22,09 & 3,71 & 21,10 & 5,60 & 20,07 & 3,19 & 24,33 & 4,12 \\
\hline IVO2 & 20,95 & 6,30 & 20,85 & 3,95 & 21,10 & 5,60 & 20,75 & 3,77 & 24,79 & 6,41 \\
\hline FVE & 21,47 & 5,46 & 25,63 & 8,49 & 22,00 & 6,48 & 29,78 & 7,25 & 35,08 & 8,33 \\
\hline TAAIL A & 48,38 & 13,65 & 45.28 & 10,30 & 46,11 & 15,00 & 42,39 & 10,02 & 38,75 & 13,44 \\
\hline ERRO A & 0.1 & & 0.3 & & $0-1$ & & 0.2 & & $0-2$ & \\
\hline TRAIL B & 104,52 & 35,45 & 110,9 & 35,48 & 109,83 & 47,87 & 78.51 & 21,98 & 78,08 & 22,38 \\
\hline ERRO B & 0.7 & & 0.5 & & 0.2 & & 0.2 & & 0.2 & \\
\hline TOKEN T. & 21,60 & 0,51 & 21 & 1,33 & 21,22 & 0,95 & 21,85 & 0,33 & 21.76 & 0,33 \\
\hline CVLT EVOC1 & 7,84 & 2,00 & 8,57 & 1,96 & 8,05 & 1,95 & 8,37 & 2,27 & 8,45 & 2,24 \\
\hline CVLT EVOC5 & 13,57 & 1,98 & 14,47 & 1,8 & 13,77 & 1,70 & 14,81 & 1,38 & 14,45 & 1,71 \\
\hline CVLT total & 57,21 & 7,06 & 61,95 & 7,56 & 60,05 & 8.73 & 63,11 & 6,95 & 61,13 & 8,82 \\
\hline CVLT ic & 12,10 & 2,80 & 13,47 & 2,42 & 11,72 & 2,42 & 13,70 & 1,68 & 13,50 & 1,62 \\
\hline CVLT icas & 13,36 & 1,89 & 14,14 & 2,00 & 13,44 & 1,97 & 14,66 & 1,46 & 14,13 & 1,28 \\
\hline CVLT il & 12,78 & 2,39 & 13.76 & 2,42 & 13,11 & 2,11 & 14,37 & 1,73 & 14,18 & 1,70 \\
\hline CVLT ilas & 13,57 & 1,80 & 14,42 & 2,29 & 13.77 & 1,83 & 14,74 & 1,37 & 14,77 & 1,23 \\
\hline CVLT rec & 15,52 & 0,96 & 15,52 & 0,81 & 15,27 & 1,07 & 15,63 & 0,62 & 15,72 & 0,55 \\
\hline S LURIA & 24,26 & 3,34 & 5,47 & 1,12 & 5,66 & 0,97 & 24,96 & 3,35 & 5,83 & 0,48 \\
\hline CUBO & 7,70 & 2,47 & 7,33 & 1,62 & 7,44 & 1,91 & 8,14 & 2,15 & 7,83 & 1,49 \\
\hline M. RAVEN & 15,88 & 3,75 & 14,04 & 4,92 & 14,77 & 3,15 & 19,59 & 4,22 & 19,18 & 5,35 \\
\hline TOL & 24,26 & 3,34 & 21,19 & 4,91 & 22,77 & 2,98 & 24,96 & 3.35 & 24,39 & 3,66 \\
\hline
\end{tabular}


(continuaçăo da pagina anterior)

\begin{tabular}{|c|c|c|c|c|c|c|}
\hline \multirow{3}{*}{$\begin{array}{l}\text { Idade } \\
\text { Escolaridade } \\
\text { Testes }\end{array}$} & \multicolumn{2}{|c|}{$15-25$} & \multicolumn{2}{|c|}{$26-35$} & \multicolumn{2}{|c|}{$36-45$} \\
\hline & \multicolumn{2}{|c|}{$>=13$} & \multicolumn{2}{|c|}{$>=13$} & \multicolumn{2}{|c|}{$>=13$} \\
\hline & Média & DP & Média & DP & Média & $\mathrm{DP}$ \\
\hline C. MENTAL & 6,71 & 1,61 & 6,90 & 1,84 & 8,00 & 0,78 \\
\hline AT & 242,85 & 42,80 & 207,40 & 45,32 & 187,06 & 41,78 \\
\hline ID & 10,24 & 7,54 & 16,64 & 10,18 & 18,47 & 14,07 \\
\hline MLOG & 12,23 & 2,07 & 12,44 & 2,60 & 12,83 & 2,65 \\
\hline MLOGL. & 11,45 & 2,69 & 11,63 & 2,57 & 11,76 & 2,54 \\
\hline PP & 18,95 & 2,28 & 19,20 & 1,70 & 19,00 & 1,94 \\
\hline PPL & 9,81 & 0,40 & 9,97 & 0,10 & 9,90 & 0,28 \\
\hline MV & 13,14 & 1,71 & 12,54 & 1,92 & 11,86 & 2,13 \\
\hline MVL & 12,16 & 2,55 & 11,86 & 2,13 & 12,13 & 2,20 \\
\hline DD & 5,95 & 1,02 & 5,76 & 1,30 & 6,26 & 1,16 \\
\hline Dinv & 5,14 & 1,10 & 5,23 & 1,44 & 5,26 & 1,53 \\
\hline STROOP C & 55,95 & 7,54 & 52,86 & 11,65 & 55,35 & 8,90 \\
\hline ERRO C & $0-2$ & & $0-2$ & & 0.2 & \\
\hline STROOP CW & 121,47 & & 126,68 & 24,28 & 138,71 & 30,83 \\
\hline ERAO CW & $0-4$ & & $0-7$ & & $0-10$ & \\
\hline IVO1 & 21,9 & 5,20 & 23,31 & 6,19 & 26,26 & 5,67 \\
\hline IVO2 & 22,95 & 5,10 & 23,40 & 4,92 & 24,20 & 4,14 \\
\hline FVE & 31,38 & 7,72 & 31,28 & 6,48 & 36,28 & 11,97 \\
\hline TAAIL A & 42,66 & 13,04 & 36,00 & 13,13 & 41,20 & 12,37 \\
\hline ERRO A & $0-2$ & & $0-8$ & & $0-1$ & \\
\hline TAAIL B & 72,57 & 24,09 & 78.72 & 20,29 & 80,13 & 20,26 \\
\hline ERRO B & $0-2$ & & $0-2$ & & 0.3 & \\
\hline TOKEN T. & 22 & 0 & 21,92 & 0.23 & 21,76 & 0.45 \\
\hline CVLT EVOC1 & 9,09 & 1,75 & 8,42 & 2,20 & 7,85 & 1,91 \\
\hline CVLT EVOCS & 15.23 & 1,37 & 14,71 & 1,34 & 15,14 & 0,94 \\
\hline CVLT total & 66,66 & 7,25 & 63.85 & 7,44 & 63,26 & 5,88 \\
\hline CVLT ic & 14 & 2,36 & 13,66 & 1,65 & 13,64 & 2,09 \\
\hline CVLT icas & 14,66 & 1,62 & 14,38 & 1,43 & 14,35 & 1,69 \\
\hline CVLT if & 14,72 & 1,70 & 13,95 & 1,71 & 14,66 & 1,34 \\
\hline CVLT ilas & 14,71 & 1,92 & 14,42 & 1,36 & 15,00 & 1,03 \\
\hline CVLT rec & 15,71 & 0,56 & 15,76 & 0,76 & 15,60 & 0.63 \\
\hline S LURIA & 5,85 & 0,67 & 5,76 & 0,7 & 5,92 & 0.26 \\
\hline CUBO & 8,25 & 0,96 & 7,90 & 1,48 & 8,21 & 1,18 \\
\hline M. RAVEN & 21,85 & 2,83 & 20,38 & 5,10 & 17,80 & 4,34 \\
\hline $\mathrm{TOL}$ & 24,85 & 2,83 & 24,85 & 3,16 & 24,06 & 3,88 \\
\hline
\end{tabular}

\section{Discussão}

Os dados de normalização da bateria de epilepsia (BE) sugerem que os factores sociodemográficos são determinantes para podermos classificar, de normal ou alterado, os resultados da avaliação neuropsicológica. Sem eles e apenas comparando os dados com os valores normativos, de populações originárias de outros países e culturas, arriscamos um parecer que pode não estar correcto.

A escolaridade influencia a forma de percepcionar, guardar e evocar. $\mathrm{O}$ treino realizado durante a aprendizagem escolar permite a aquisição de estratégias que nos conduzem a melhor aproveitamento de informação e menor dispêndio de energia. Esse efeito manifesta-se em provas verbais mas também em não verbais, ao contrário do que afirma Botwinick (1977). Dezassete em 
trinta e cinco variáveis têm características de realização que dependem da aprendizagem escolar.

A influência da escolaridade nas provas da EMW é conhecida, mas o resultado da CVLT total, que habitualmente não revela alterações (Delis et al., 1987), também se modifica segundo o nível de literacia. Estes dados sugerem que o grupo mais literado conseguiu desenvolver estratégias que lhe permitem, rapidamente, guardar maior quantidade de informação. A mais facilmente evidenciada é a de organização por campos semânticos, que nos leva a concluir que a utilização da organização/memória semântica é um processo cerebral elaborado, que se desenvolve através de estimulação e aprendizagem. As provas de iniciativa verbal, semântica e fonológica, que também dependem da memória, mostraram igual transformação.

Também o processamento de material não verbal pode ser melhorado pela literacia. Segundo Ardila $(1989,1993)$, os indivíduos com maior contacto com a natureza têm estratégias visuoespaciais mais desenvolvidas que os da cidade. Poder-se-ia então pensar que a escolaridade teria pouca importância no resultado. O que se verifica é que as capacidades necessárias para a realização destas provas consistem em métodos correntemente usados no dia-a-dia da nossa sociedade e aprendidos na escola, por isso a influência mantém-se.

Apesar de alguns autores anglos-axónicos (Dodrill, 1989; Saykin et al., 1995) não ajustarem os valores em função da idade, argumentando que no intervalo que considerámos não existem perdas ou alterações significativas, a análise da nossa população demonstra o contrário. Os resultados dos testes têm valores inversamente proporcionais ao grupo etário. Os testes mais afectados pela idade foram os índices de atenção RT e ID, o tempo de realização do Stroop CW, o desenho do cubo e dois subtestes da EMW (PP e MVL).

Estes valores, segundo outros autores, começam a declinar entre os 30 e os 50 anos (Albert \& Naeser, 1987), sendo a memória visual a que mais precocemente é afectada. A evocação imediata e a tardia após intervalo de latência, aumentam a sensibilidade do teste com diferenças intragrupo maiores em populações com uma média de idades mais elevada (Ardila \& Rosseli, 1989).

Nas provas em que o tempo é cronometrado também se nota um declínio dos resultados, provavelmente por maior lentificação geral (Van Gorp \& Staz, 1990).

O melhor desempenho nas MRaven, pelos homens mais jovens e com escolaridade mais elevada, espelha a natureza do teste. A sua realização parece depender da aprendizagem e treino de conceitos, ainda que não verbais, que estão relacionados com o número de anos de escolaridade, com o tipo de treino e com a proximidade no tempo do mesmo. Na literatura internacional não estão descritas influências tão marcadas das variáveis sociodemográficas mas é provável que o nosso tipo de cultura saliente estes aspectos.

Os resultados relativos à influência do sexo são concordantes com os da literatura. Na nossa amostra as provas MRaven e LOT foram melhor desempenhadas pelos indivíduos do sexo masculino, enquanto que as mulheres 
obtiveram melhores resultados no CVLT. Em cinco factores do CVLT estas conseguiram melhores cotaçōes: CVLT5; CVLT ic; CVLT icas; CVLTil; CVLTtotal. Por outro lado, também foi descrito (6) o melhor desempenho do TOL pelos homens.

Quer o TOL quer o CVLT são testes nos quais já era conhecida a influência da variável sexo (Lezak, 1995; Delis et al., 1987). Em relação às MRaven não é referida na literatura qualquer variabilidade nesse sentido mas, na nossa amostra, os melhores resultados foram conseguidos pelos homens jovens com escolaridade mais elevada. Devido a não ser suficiente o tamanho da amostra do grupo de controlo não puderam ser criados subgrupos que permitissem analisar os dados em relação a subgrupos opostos. $\mathrm{O}$ melhor desempenho desta tarefa pelos elementos deste grupo revela a complexidade que implica a tarefa de categorizar material não verbal. A aprendizagem e treino recentes, de conceitos, parecem ser determinantes. As diferenças anatómicas, culturais, étnicas e educacionais existem e estão presentes na estrutura e função cerebral dos humanos.

Em conclusão, através dos resultados apresentados podemos avaliar a importância da adaptaçăo e normalização de uma bateria de testes na população portuguesa. Mesmo testes que em outras culturas não evidenciam notória influência das características sociodemográficas, revelam na nossa populaçăo uma maior susceptibilidade, demonstrando a necessidade de a estratificar.

\section{Notas}

1 A autora agradece a Prof." Dra. Isabel Pavão e ao Prof. Dr. Alexandre Castro-Caldas a revisāo do artigo. A avaliaçāo dos individuos do grupo de controlo foi em parte financiada pela Bolsa Tecnifar 1994.

\section{Referências}

Albert M., Duffy F. H., \& Naeser, M. (1987). Nonlinear changes in cognition with age and their neuropsychological correlates. Canadian Journal of Psycholology., 41, 141-157

Amaral, J. R. (1966). Aferi̧̧ð̆o do teste LA. Coimbra: Publicaçōes da Universidade de Coimbra.

Ardila, A. (1993). Historical evolution of spatial abilities. Behavioral Neurology,6, 83-87.

Ardila A., \& Rosseli, M. (1989). Neuropsychological characteristics of normal aging. Developmental Neuropsychology., 5, 307-20.

Ardila A., Rosselli, M., \& Rosas, P. (1989). Neuropsychological assessment of illiterates: visuospatial and memory abilities. Brain \& Cognition, 11 (2), 147-166. 
Aria, A. (1958), Elementi di psicologia clinica. Torino: Minerva Medica.

Benton, A. L., Hamsher, K. S., Varney, N. R., \& Spreen, O. (1983). Judgement of line orientation form $h$. contributions to neuropsychological assessment. Nova Iorque: Oxford University Press.

Botwinick, J. (1977). Intellectual abilities. In J. E. Birren \& Schaiekw (Eds.), Handbook of the psychology of aging (pp. 550-650). Nova Iorque: Van Nostrand.

Castro-Caldas, A. (1979). Diagnóstico e evolução das afasias de causa vascular. Dissertação de doutoramento, Faculdade de Medicina de Lisboa.

Christensen, A. L. (1975). Luria neuropsychological investigation text. Nova Iorque: Spectrum Publications.

Damásio, A. R. (1973). Perturbaçð̄es neurológicas da linguagem e de outras fuņ̣̄és simbólicas. Dissertação de doutoramento, Faculdade de Medicina de Lisboa.

De Renzi, \& Vignolo, L. (1962). The Token test: A sensitive test to detect receptive disturbances in aphasia. Brain, 85, 665-678.

Delis, D. C., et al. (1987). California verbal learning test. adult version research edition manual. San Antonio: The Psychological Corporation.

Dodrill, C. B. (1978). A neuropsychological battery of epilepsy. Epilepsia, 19, 611-23.

Ferro, J. M. (1986). Neurologia do comportamento. Dissertação de doutoramento, Faculdade de Medicina de Lisboa.

Frisk, V., \& Milner, B. (1990). The relationship of working memory to the immediate recall of stories following unilateral temporal or frontal lobotomy. Neuropsychologia, 28, 121-135.

Golden (1978). Stroop color and word test. Chicago: Stoelting.

Heaton, R. K. (1981). Wisconsin card sorting test. manual odessa Florida. Psychological Assessment Resources.

Ivnik, R. J. (1987). Memory testing. In T. Yanagihara \& R. C. Petersen (Eds.), Memory disorders, research and clinical practice. Nova Iorque: Marcel Dekker.

Jokeit, H., Heger, R., Ebner, A., Markowitsch, H. J. (1988). Hemispheric asymmetries in category-specific word retrivial. Neuroreport, 13, 9 (10), 2371-2373.

Jones-Gotman, M. (1991). Localization of lesions by neuropsychological testing. Epilepsia. S41-S52.

Klove H. (1963). Clinical Neuropsychology. In F. M. Forster (Ed), The medical clinics of North America. Nova Iorque. Saunders. 1963.

Lezak, M. D. (1995). Neuropsychological assessment (2." ed.). Nova Iorque: Oxford University Press.

Luria, A. R. (1965). Neuropsychological analysis of focal brain lesions. In B. B. Wolman (Ed.), Handbook clinical psychology. Nova Iorque: McGraw Hill.

Miles, W. R. (1980). Test ABC de domináncia ocular. Madrid: Publicaciones de Psicologia Aplicada.

Milner, B. (1967). Brain mechanisms suggested by studies of temporal lobes. In C. H. Millikan \& F. L. Darley (Eds.), Brain mechanisms underlying speech and language. Nova lorque: Grune \& Stratton, .

Minden, S. L., Moes, E. J., Orav, J., et al. (1990). Memory impairment in multiple sclerosis. Journal of Clinical and Experimental Neuropsychology, 12, 566-586

Oldfield, R. C. (1971). The assessment and analysis of handedness: The Edinburgh inventory. Neuropsychologia, 97-113. 
Rausch, R., L-M., \& Langfitt, J. (1998). Neuropsychological evaluation - adults. In J. Engel \& T. Pedley (Eds.), Epilepsy: A comprehensive textbook (vol. 1). Filadélfia: Lippincott-Raven Publishers.

Raven (1938). Progressive Matrices: A perceptual test of intelligence. Londres: H. K. Lewis.

Reitan, R., \& Wolfson, D. (1985). The Halstead-Reitan Neuropsychological Battery: Theory and clinical interpretation. Arizona: Neuropsychological Press.

Reitan, R. M., \& Davison, L. A. (1974). Clinical neuropsychology: Current status and applications. Nova Iorque: HV. Winston.

Russel, E. W. (1975). A multiple scoring method for assessment of complex memory functions. Journal of Consulting and Clinical Psychology, 3, 800-809.

Saykin, A. J., Gur. C., Gur. R., et al. (1995). Normative neuropsychological test performance: Effects of age, education, gender and ethnicity. Applied Neuropsychogyl, 2, 79-88.

Spreen, O., \& Strauss, E. (1987). A compendium of neuropsychological tests. Nova Iorque: Oxford University Press.

SPSS for Windows. Release 8.00. (1997). Standard version. Spss Inc.

Strub, R. L, \& Black, F. W. (1977). The mental status examination in neurology. Filadelfia: Davis F. A. Company.

Thurstone, L. L., \& Thurstone, T. G. (1962). Primary mental abilities. Chicago: Science Research Associates.

Trahan, D. E., Quintana, J., Willinghan, A. C., \& Goethe, K. E. (198). The visual reproduction subtest standardization and clinical validation of a delayed recall procedure. Neuropsychology., 2, 29-39.

Van Gorp, W. G., \& Mahler, M. Subcortical features of normal aging. In J. Cummings (Ed.), Subcortical dementia. Nova Iorque: Oxford University Press.

Van Gorp, W. G., Satz, P., \& Mitrushoma, M. (1990). Neuropsychological processes associated with normal aging. Developmental Nerurospsychology., 6, 279-90.

Wechsler, D. A. (1945). Standardized memory scale for clinical use. Journal of Psychology, 19, 87-95.

Neuropsychological evaluation is essential for epileptic patient diagnosis and treatment (abstract) In order to create a battery adequated to Portuguese epileptic patients with epilepsy secondarily to temporal or frontal foci, we elaborated a battery of selected cognitive tests. A control group of one hundred and sixty healthy individuals were submitted to this battery in order to collect normative data. Results were analysed according to age, literacy and sex and confirm demographic influences in several tasks. Comparison of groups revealed variation of capacities both on verbal and visual tasks. These findings show the critical importance of neuropsychological tests normalization so as to clearly determine the meaning of results. 